\title{
How do fits of simulated magnetic clouds correspond to their real shapes in 3-D?
}

\author{
M. Vandas ${ }^{1}$, E. Romashets ${ }^{2,3}$, and A. Geranios ${ }^{4}$ \\ ${ }^{1}$ Astronomical Institute, Academy of Sciences of the Czech Republic, 14131 Praha 4, Czech Republic \\ ${ }^{2}$ Prairie View A\& M University, Mail Stop 2250, P.O. Box 519, Prairie View TX 77446, USA \\ ${ }^{3}$ Institute of Terrestrial Magnetism, Ionosphere, and Radio Wave Propagation, Russian Academy of Sciences, Troitsk, \\ Moscow Region, 142092, Russia \\ ${ }^{4}$ Physics Department, University of Athens, Panepistimioupoli-Kouponia, Athens 15771, Greece
}

Received: 29 May 2009 - Revised: 25 January 2010 - Accepted: 11 August 2010 - Published: 26 August 2010

\begin{abstract}
Magnetic clouds are important objects for space weather forecasters due to their impact on the Earth's magnetosphere and their consequences during geomagnetic storms. Being considered as cylindrical or toroidal flux ropes, their size, velocity, magnetic field strength, and axis orientation determine its impact on Earth. Above mentioned parameters are usually extracted from model fits using measurements from one-spacecraft crossings of these structures. In order to relate solar events with these spacecraft observations, the parameters are then compared to situation at the Sun around a most probable source region with a goal to correlate them with near-Sun observed quantities for prediction purposes. In the past we performed three-dimensional simulations of magnetic cloud propagation in the inner heliosphere. Simulated spacecraft measurements are fitted by models of magnetic clouds and resulting parameters are compared with real shapes of magnetic clouds which can be directly obtained from our simulations. The comparison shows that cloud parameters are determined quite reliably for spacecraft crossings near the cloud axis.
\end{abstract}

Keywords. Interplanetary physics (Interplanetary magnetic fields) - Solar physics, astrophysics, and astronomy (Flares and mass ejections) - Space plasma physics (Numerical simulation studies)

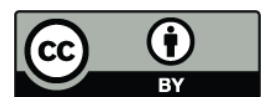

Correspondence to: $\mathrm{M}$. Vandas (vandas@ig.cas.cz)

\section{Introduction}

Magnetic clouds were discovered in solar wind observations as regions with an enhanced magnetic field magnitude, smooth rotation of magnetic field vector, decreased proton temperature, and size of the order of $0.1 \mathrm{AU}$ (Klein and Burlaga, 1982). The regions are interpreted as manifestations of large interplanetary flux ropes, probably still magnetically connected to the solar corona by their feet when they are registered at $1 \mathrm{AU}$.

There are numerous models which try to model observed magnetic clouds as flux ropes, to compare measurements with model profiles, and thus to extract some basic parameters of these phenomena (e.g., Marubashi, 1986, 1997; Burlaga, 1988; Lepping et al., 1990, 2006; Hidalgo et al., 2000, 2002; Mulligan and Russell, 2001; Hu and Sonnerup, 2001; Vandas et al., 2005, 2006; Dasso et al., 2006; Marubashi and Lepping, 2007; Nakwacki et al., 2008). Most models assume a cylindrical geometry with quantities not changing along the axis (i.e., a two-dimensional problem). There are specific assumptions on magnetic fields (e.g., a force-free field) and temporal behaviour (e.g., stationarity or radial expansion). Models yield basic parameters of the flux ropes as their radius, value of the axial magnetic field, chirality, or helicity per unit length. One can think about how these parameters are real or reliable, because they are mostly extracted from single spacecraft crossings, i.e., from measurements along only a single line through a flux rope. It is difficult to verify independently obtained geometric parameters of the cloud like its axis orientation or its dimensions, because we do not "see" needed quantities apart from the trajectory. Simultaneous crossings of magnetic clouds by more

Published by Copernicus Publications on behalf of the European Geosciences Union. 


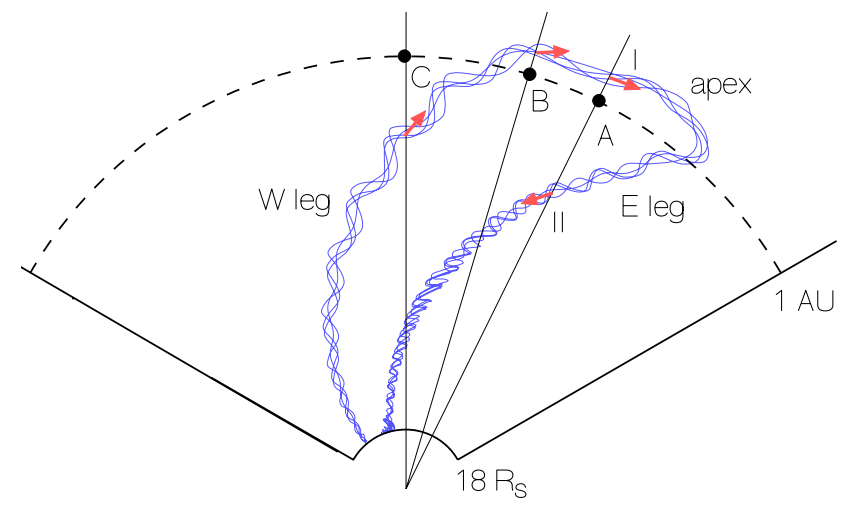

Fig. 1. Simulated flux rope lying in the ecliptic plane, after it reached $1 \mathrm{AU}$. Thin helical lines in blue are magnetic field lines of the flux rope. Parts of the flux rope are labeled as apex, W (west) leg, and E (east) leg. The thick solid lines are boundaries of a computational domain ( $R_{\mathrm{S}}$ means the solar radius), the dashed arc shows the distance of $1 \mathrm{AU}$. A more detailed description is in the text.

spacecraft sufficiently separated are rare and so are papers analyzing them (e.g., Mulligan and Russell, 2001; Liu et al., 2008; Möstl et al., 2008; Kilpua et al., 2009).

On the other hand, magnetohydrodynamic simulations of magnetic cloud propagation (e.g., Vandas et al., 2002; Odstrcil et al., 2002; Manchester et al., 2004, Chané et al., 2006; Jacobs et al., 2009) have the advantage that necessary quantities are known globally and such that simulations can provide what a spacecraft can measure at given points. Therefore, they are suitable for testing fitting procedures. One may admit that simulations are simpler than probably the real phenomena are. But even in this case they provide us with basic information on credibility of fittings.

A comparison of fits with one simulated case of a flux rope from two-dimensional simulations has been presented by Riley et al. (2004). Simulated measurements were provided to several groups, which are involved in fitting of observed magnetic clouds, and they independently determined basic parameters (without knowing details of the simulation). The authors conclude that accuracy of model parameters decreases markedly with increasingly glancing encounters and that correct identification of the boundaries of the flux rope can be a significant limiter.

Here we present comparisons between fits and real situations from three-dimensional simulations. Magnetic clouds in the simulations are truly three-dimensional bodies, which have a form of loop-like flux ropes that are connected by both feet to the Sun. The paper by Riley et al. (2004) treated one case: a flux rope parallel to the ecliptic plane (with inclination $0^{\circ}$ in our nomenclature, the limitation given by their 2.5-D approach). In our more general approach, we deal here with many cases, flux ropes with various inclinations that are crossed in various parts of their loop-like bodies. The simu- lated flux rope in the Riley et al. (2004) paper has an extreme oblate shape which can be hardly fitted by existing models. Our simulations yielded moderate oblate shapes which are closer to assumptions of current models (circular/elliptic shape). Reasons for this distinct flux rope shape difference in simulations are discussed in the Discussion and conclusions section. In addition and, unlike the Riley et al. (2004) paper, we provide a direct visual inter-comparison between real and modelled flux rope shapes.

\section{Simulations}

Three-dimensional simulations of a flux rope propagation in a simple solar wind were performed for various flux rope orientations. The simulations used one-fluid time-dependent ideal-MHD equations. The simulations and their results have been described in Vandas et al. (2002, 2003). The simple quiet solar wind model contained no magnetic sectors and no heliospheric current sheet, the radial velocity only depended on the radial distance. The flux rope was introduced into the computational domain by perturbations of quantities at the inner boundary. A model of the flux rope was a toroid with a constant $\alpha$ force-free field inside it. During injection of the toroid, only the magnetic field was perturbed at the inner boundary; other quantities remained unchanged. When a half of the toroid emerged, its feet were kept at the inner boundary and shifted westward to simulate their solar rotation. Magnetic fields have a lower level than in a real solar wind in order to suppress numerical reconnection (Vandas et al., 2002). Here these results are used to test our fitting procedure. Flux ropes in simulations had inclinations to the ecliptic plane of $0^{\circ}, 20^{\circ}, 45^{\circ}, 70^{\circ}$, and $90^{\circ}$. The first case $\left(0^{\circ}\right)$ was studied the most and it is displayed in Fig. 1.

Figure 1 shows a simulated flux rope (magnetic cloud) propagating in the ecliptic plane, after it reached $1 \mathrm{AU}$. Its leading part (apex) is rather flat and its legs are curved approximately as Archimedean spirals of the surrounding magnetic field. Hypothetic spacecraft were introduced into our computational domain at $1 \mathrm{AU}$ in three places, labeled $\mathrm{A}$, B, and C (see black bullets in Fig. 1). These spacecraft "recorded" plasma and magnetic field parameters in a time sequence. Obtained "measurements" were then fitted by a flux rope model. Figure 1 also shows estimated flux-ropeaxis orientations by red arrows; the estimations were simply done from the figure by eye. In the point A the spacecraft observed the flux rope two times within our simulation period, which are denoted as events I and II. Radial directions connecting the three points with the Sun are also plotted.

\section{Flux-rope model}

Our flux-rope model assumes a constant-alpha force-free magnetic structure inside a cylinder (which can be elliptical) and includes a flux-rope radial expansion. Amount of 
(c)

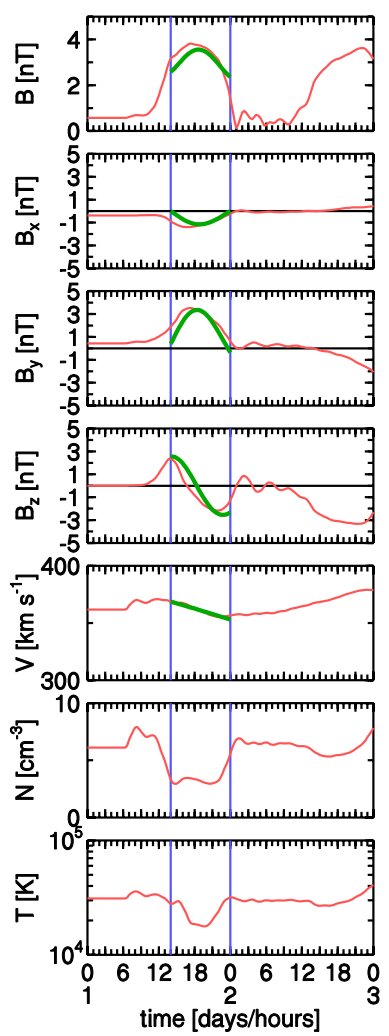

(s)

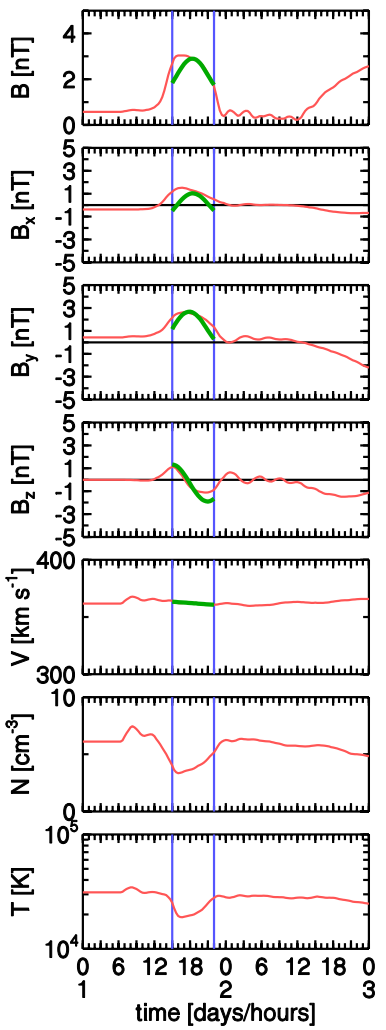

Fig. 2. Simulated time profiles (red) in the point A (c) and a point slightly above it (s) for the event I. The plot displays from top to bottom the magnetic field magnitude $B$, magnetic field components $B_{\mathrm{X}}, B_{\mathrm{y}}$, and $B_{\mathrm{Z}}$ (in GSE), velocity magnitude $V$, number density $N$, and temperature $T$. Vertical blue lines denote estimated flux-rope boundaries. Model fits are plotted by the thick green lines.

expansion is estimated from an observed velocity slope. The orientation of the flux-rope axis is given by two angles, the inclination $\theta_{\mathrm{c}}$ and the azimuthal angle $\varphi_{\mathrm{c}}$ (in GSE system). Here these angles are determined from variance analysis of "observed" magnetic field vectors (Lepping et al., 1990), that is, in an objective way. Our model is described in Vandas et al. (2006). It is a generalization of the Lundquist solution; the latter is commonly used as a model for magnetic cloud fits (Burlaga, 1988; Lepping et al., 1990, 2006). The generalization includes oblateness and expansion of the magnetic structure. Oblateness is conserved during expansion, which is incorporated by time-dependent $\alpha$ and axial magnetic field parameters.

\section{Results}

Simulated magnetic field components and velocity magnitude profiles in given points from Fig. 1 (i.e., what a hypothetic spacecraft would measure, e.g., in point A) were fitted

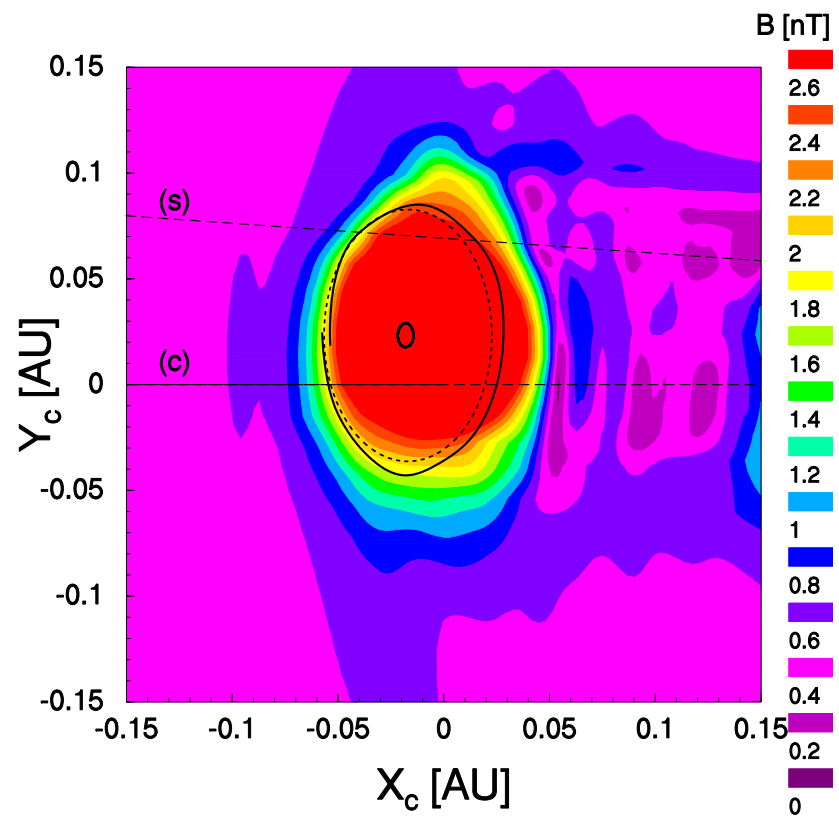

Fig. 3. Cross section through the flux rope around the point A for the event I. Contours show the distribution of the magnetic field magnitude. Thick ovals are projected parts of magnetic field lines. Dashed straight lines are projected trajectories of hypothetic spacecraft. $X_{\mathrm{c}}$ and $Y_{\mathrm{c}}$ are axes of a local Cartesian coordinate system with the $Z_{\mathrm{c}}$ axis along the estimated flux rope axis (the red arrow from Fig. 1). So the $X_{\mathrm{c}}$ and $Y_{\mathrm{c}}$ axes lie in the plane perpendicular to the flux rope axis, but their orientation in this plane is selected arbitrarily.

by our model. The parameters of flux ropes were obtained by an objective procedure, which finds ones with the least mean square difference between observed and modeled profiles. Then the model flux rope (its cross section) is compared with the "true"one from MHD simulations.

Simulated measurements of a flux rope lying in the ecliptic plane (inclination $0^{\circ}$, Fig. 1) are analyzed first (cases 1-4): an apex of the flux rope at the point $A$ (case 1), a leg of the flux rope at the point A (case 2), an apex at the point B (case 3), and a leg at the point C (case 4). An apex of flux ropes in various nonzero inclinations at the point $\mathrm{A}$ was then treated as cases 5-8. A detailed description of case 1 is given in Sect. 4.1 and Figs. 2 and 3. Case 2 is presented in Sect. 4.2; and case 3, in Sect. 4.3 and Fig. 4. Case 4 is discussed in Sect. 4.4 and Figs. 5-6. Cases 5-8 are presented in Sect. 4.5 and demonstrated in Figs. 7-9. The results are summarized in Table 1 and in the Discussion and conclusions section.

\subsection{Point A - event I ( case 1)}

Figure $2 \mathrm{c}$ shows simulated time profiles for the point $\mathrm{A}$ and the first flux-rope crossing (crossing of the apex, event I; the (c) refers to panels on the left side of Fig. 2). Vertical lines 


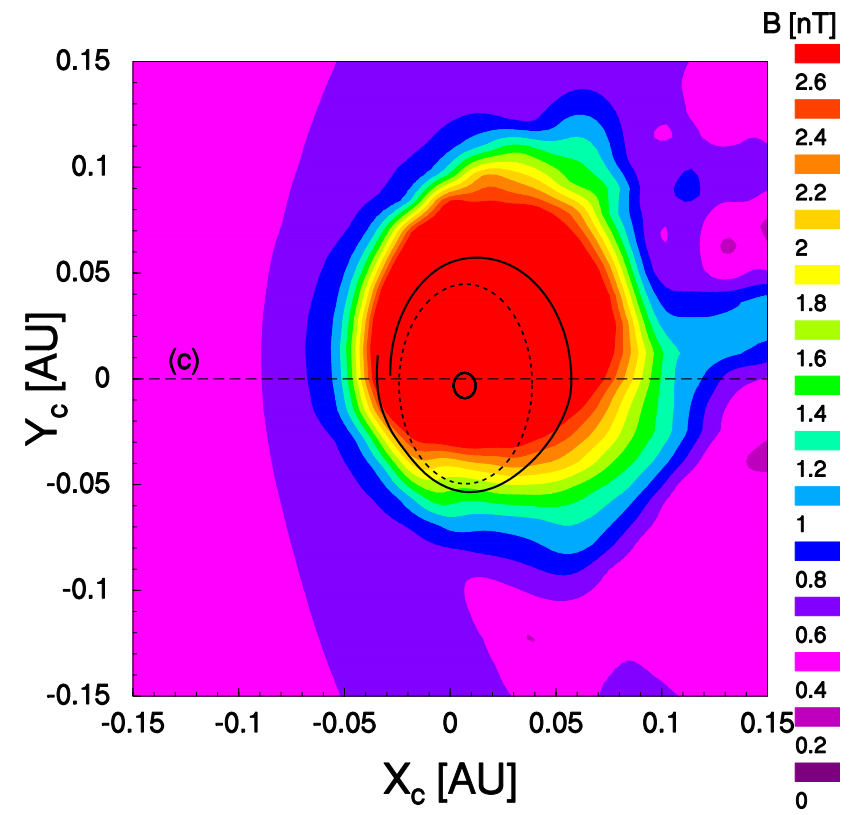

Fig. 4. Cross section through the flux rope around the point B. Outline is similar to Fig. 3.

denote estimated flux-rope boundaries. The estimation is based on temperature and density drops. These "measured" profiles show clear signatures of a magnetic cloud: a magnetic field magnitude increase, rotation of the magnetic field vector (sinusoidal profiles of magnetic field components), and a temperature decrease. A second $B$-increase, which is cut at the right, is the event II.

A cross section through the flux rope around the point $\mathrm{A}$ is shown in Fig. 3. Thick ovals denote a shape of the rope and a position of its axis. However, a real extent of the flux rope is slightly larger than the greater oval. The dashed line labeled (c) is a projected trajectory of the hypothetic spacecraft in the point A. The profiles in Fig. 2c correspond to this trajectory. It is called a central crossing as it goes closer to the axis.

The second dashed trajectory ( $\mathrm{s}$ ) is from a spacecraft near the point A but slightly above the ecliptic plane. It is called a side crossing, because it has a larger impact factor $p$. The impact factor is a relative minimum distance from the rope axis, the distance divided by the rope radius $r_{0}$ (for an oblate rope it is divided by the minor radius). "Measurements" along this trajectory are shown in Fig. 2s.

Estimated flux rope orientation (the arrow in Fig. 1) has $\theta_{\mathrm{c}}=0^{\circ}$ and $\varphi_{\mathrm{c}}=95^{\circ}$. "Measurements" displayed in red in Fig. 2 were fitted by the model mentioned above and the fits are shown by the thick green lines in this figure. Determined flux-rope parameters are for fit $(\mathrm{c}): \theta_{\mathrm{c}}=-8^{\circ}$, $\varphi_{\mathrm{c}}=109^{\circ}, p=0, r_{0}=0.04 \mathrm{AU}$, oblateness $=1.5$; and for fit (s): $\theta_{\mathrm{c}}=-8^{\circ}, \varphi_{\mathrm{c}}=32^{\circ}, p=0.5, r_{0}=0.02 \mathrm{AU}$, oblateness $=1.0$. Oblateness is the ratio of major to minor radii for
Table 1. True and modeled parameters of magnetic flux ropes. The lines, where the trajectory column (tra) is marked by "-", give true parameters ("c" and "s" mean central and side crossings, respectively). The $r_{0}$ is in AU, $o$ is oblateness $\left(a / b\right.$ where $\left.b=r_{0}\right)$. Undetermined or non-reliable parameters are labeled by ?, the $p$ column shows true values in parentheses.

\begin{tabular}{cccrrccc}
\hline case & point & tra & $\theta_{c}$ & $\varphi_{c}$ & $r_{0}$ & $o$ & $p$ \\
\hline 1 & A-I & - & $0^{\circ}$ & $95^{\circ}$ & 0.04 & 1.6 & - \\
& & $\mathrm{c}$ & $-8^{\circ}$ & $109^{\circ}$ & 0.04 & 1.5 & $0.0(0.5)$ \\
& & $\mathrm{s}$ & $-8^{\circ}$ & $32^{\circ}$ & 0.02 & 1.0 & $0.5(1.2)$ \\
2 & \multirow{2}{*}{ A-II } & - & $0^{\circ}$ & $315^{\circ}$ & $?$ & $?$ & - \\
& & $?$ & $-6^{\circ}$ & $289^{\circ}$ & $?$ & $?$ & $?$ \\
3 & B & - & $0^{\circ}$ & $110^{\circ}$ & 0.04 & 1.3 & - \\
& & $\mathrm{c}$ & $9^{\circ}$ & $105^{\circ}$ & 0.03 & 1.5 & $0.0(0.1)$ \\
4 & $\mathrm{C}$ & - & $0^{\circ}$ & $140^{\circ}$ & 0.06 & 1.1 & - \\
& & $\mathrm{c}$ & $-2^{\circ}$ & $143^{\circ}$ & 0.05 & 1.5 & $0.0(0.0)$ \\
& & $\mathrm{s} 1$ & $-6^{\circ}$ & $76^{\circ}$ & $?$ & $?$ & $(0.9)$ \\
& & $\mathrm{s} 2$ & $9^{\circ}$ & $134^{\circ}$ & $?$ & $?$ & $(0.9)$ \\
5 & A-I & - & $-20^{\circ}$ & $95^{\circ}$ & 0.04 & 1.5 & - \\
& & $\mathrm{c}$ & $-17^{\circ}$ & $79^{\circ}$ & 0.04 & 1.5 & $0.0(0.3)$ \\
& & $\mathrm{s}$ & $-17^{\circ}$ & $116^{\circ}$ & 0.04 & 1.5 & $0.5(1.5)$ \\
6 & A-I & - & $-45^{\circ}$ & $147^{\circ}$ & $?$ & $?$ & - \\
& & $?$ & $-56^{\circ}$ & $93^{\circ}$ & $?$ & $?$ & $?$ \\
7 & A-I & - & $-70^{\circ}$ & $147^{\circ}$ & $?$ & $?$ & - \\
& & $?$ & $-72^{\circ}$ & $117^{\circ}$ & $?$ & $?$ & $?$ \\
8 & A-I & - & $-90^{\circ}$ & - & $?$ & $?$ & - \\
& & $?$ & $-83^{\circ}$ & - & $?$ & $?$ & $?$ \\
\hline
\end{tabular}

an elliptic cross section. The central crossing yields quite plausible parameters (except of $p$ ), the resulting shape of the model rope is shown in Fig. 3 by a dashed oval. The side crossing gives wrong parameters and the fit is worse. It was found that the fitting procedure was not very sensitive to values of oblateness and impact parameter. Therefore we use only rough characteristic values in our analysis: $0,0.25,0.5$, 0.75 for $p, 0,1.5,2,3$ for oblateness, and selected the best fit among them.

\subsection{Point A - event II (case 2)}

This event is the second flux-rope crossing for the point A, central crossing of the east leg. Estimated axis orientation (the arrow in Fig. 1) is $\theta_{\mathrm{c}}=0^{\circ}$ and $\varphi_{\mathrm{c}}=315^{\circ}$. Variance analysis yielded $\theta_{\mathrm{c}}=-6^{\circ}$ and $\varphi_{\mathrm{c}}=289^{\circ}$. The correspondence is quite good. Global data were not available for this crossing, so comparison of geometric parameters was not done. A side crossing was too far from the axis and did not show a clear signature of a flux rope crossing.

\subsection{Point B (case 3)}

A cross section through the flux rope near the point $\mathrm{B}$ is shown in Fig. 4. The hypothetic spacecraft made a central crossing. Estimated axis orientation (the arrow in Fig. 1) 
(c)
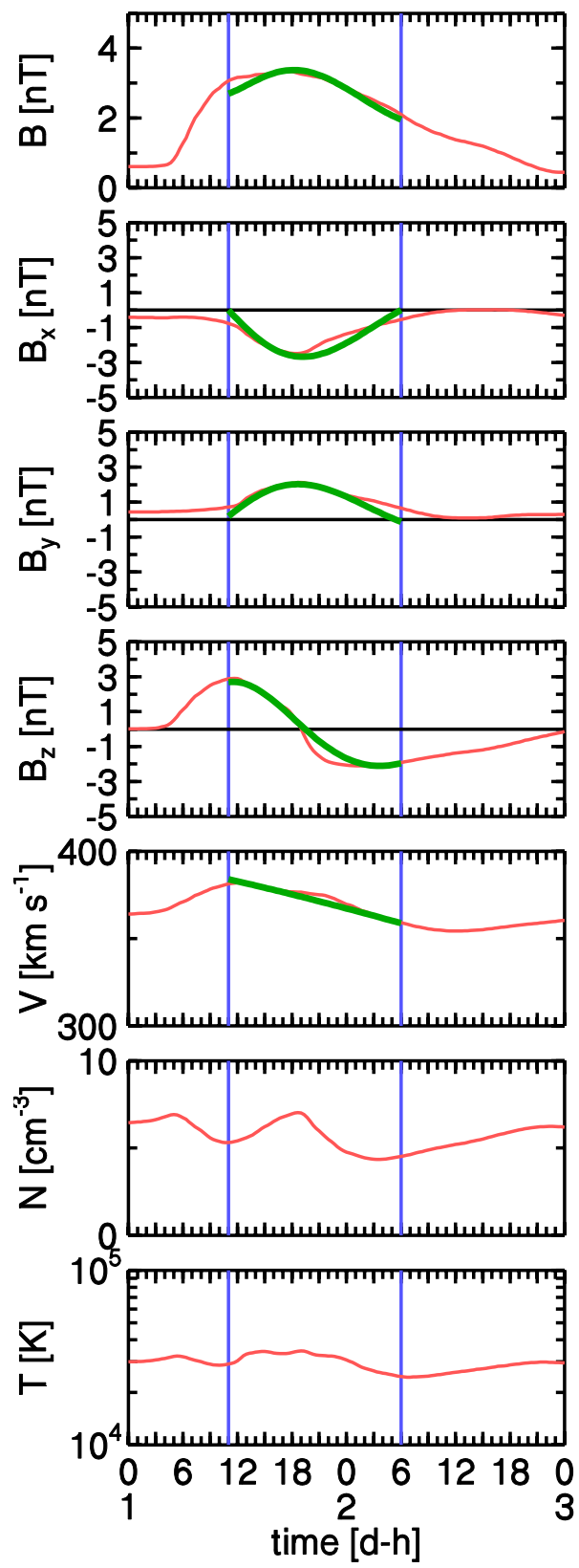

Fig. 5. Simulated time profiles in the point $C$. The layout is similar to Fig. 2.

is $\theta_{\mathrm{c}}=0^{\circ}$ and $\varphi_{\mathrm{c}}=110^{\circ}$. Determined flux-rope parameters from a fit are: $\theta_{\mathrm{c}}=9^{\circ}, \varphi_{\mathrm{c}}=105^{\circ}, p=0, r_{0}=0.03 \mathrm{AU}$, oblateness $=1.5$. The shape of the rope according to this fit is shown as a dashed oval in Fig. 4.

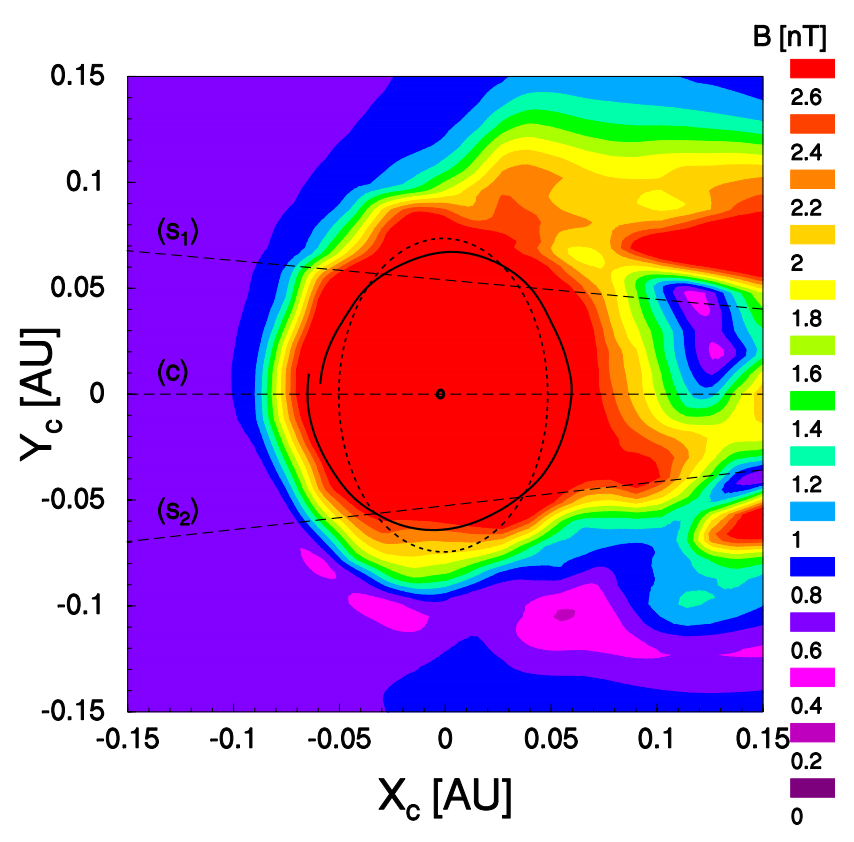

Fig. 6. Cross section through the flux rope around the point $\mathrm{C}$. Outline is similar to Fig. 3.

\subsection{Point $\mathrm{C}$ (case 4)}

Figure $5 \mathrm{c}$ shows simulated time profiles for the point $\mathrm{C}$, a central crossing of the west leg. The fit is quite good in this case, the best from all fits presented here.

A cross section of a part of the flux rope which passes near the point $\mathrm{C}$ is shown in Fig. 6. Three dashed lines are projected trajectories of hypothetic spacecraft for central (c) and two side $\left(\mathrm{s}_{1}, \mathrm{~s}_{2}\right)$ crossings. Estimated axis orientation (the arrow in Fig. 1) is $\theta_{\mathrm{c}}=0^{\circ}$ and $\varphi_{\mathrm{c}}=140^{\circ}$. A fit yielded for the central crossing (c): $\theta_{\mathrm{c}}=-2^{\circ}, \varphi_{\mathrm{c}}=143^{\circ}, p=0$, $r_{0}=0.05 \mathrm{AU}$, oblateness $=1.5$. The model shape is shown in Fig. 6 as a dashed oval. Despite of the fact that the fit of profiles is good, the shapes do not correspond so well; the real shape is close to a circle. Leg events are not "classical" magnetic clouds, temperature is increased in them (see discussion in Vandas et al., 2002). The side crossings did not give reasonable results except of axis orientation from variance analysis. Fit $\left(\mathrm{s}_{1}\right): \theta_{\mathrm{c}}=-6^{\circ}, \varphi_{\mathrm{c}}=76^{\circ}$; fit $\left(\mathrm{s}_{2}\right): \theta_{\mathrm{c}}=9^{\circ}$, $\varphi_{\mathrm{c}}=134^{\circ}$.

\subsection{Point A for inclined flux ropes (cases 5-8)}

Figure 7s shows simulated time profiles for the point A (side crossing) and Fig. 7c shows them for a site near the point A (central crossing), but for a flux rope inclined $20^{\circ}$ to the ecliptic plane.

A cross section through this inclined flux rope near the point A is shown in Fig. 8 together with trajectories of the central and side crossings. Estimated axis orientation (from 
(c)

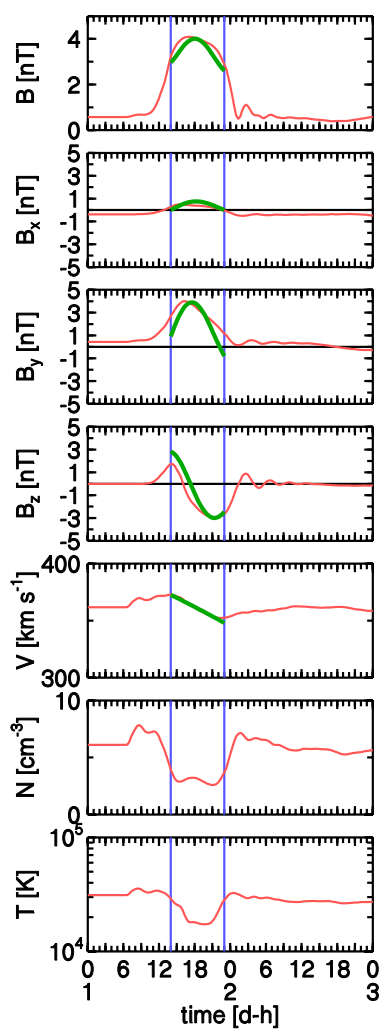

(s)

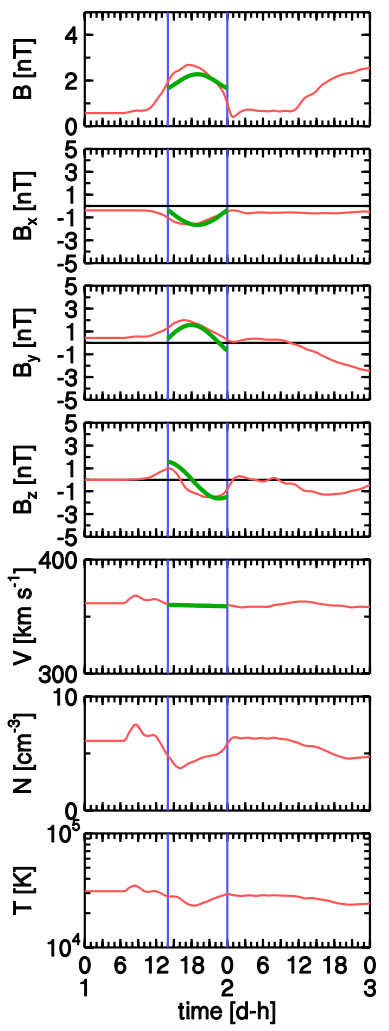

Fig. 7. Simulated time profiles near (c) and in the point A (s) for a flux rope inclined $20^{\circ}$ to the ecliptic plane. The layout is similar to Fig. 2.

analogy with the case I): $\theta_{\mathrm{c}}=-20^{\circ}, \varphi_{\mathrm{c}}=95^{\circ}$. Determined flux-rope parameters from a model fit (c): $\theta_{\mathrm{c}}=-17^{\circ}, \varphi_{\mathrm{c}}=$ $79^{\circ}, p=0, r_{0}=0.04 \mathrm{AU}$, oblateness $=1.5$; fit (s): $\theta_{\mathrm{c}}=-17^{\circ}$, $\varphi_{\mathrm{c}}=116^{\circ}, p=0.5, r_{0}=0.04 \mathrm{AU}$, oblateness $=1.5$. In this case, the both crossings yield quite plausible parameters.

Comparisons of estimated flux-rope axis orientations from geometric considerations and that from variance analysis of simulated profiles for other three inclinations, as well as the above described results, are given in Table 1. Figure 9 shows simulated time profiles (red) at the point A for case 8, a flux rope inclined $90^{\circ}$ to the ecliptic plane, and their fits (green) by the model.

\section{Discussion and conclusions}

Three-dimensional simulations of magnetic cloud propagation in the inner heliosphere were used to verify fits by our model of a magnetic cloud. The study led us to the following conclusions. Central crossings yield quite plausible determination of the axis orientation and the rope radius. It is important to point out that this is not true for side crossings. The

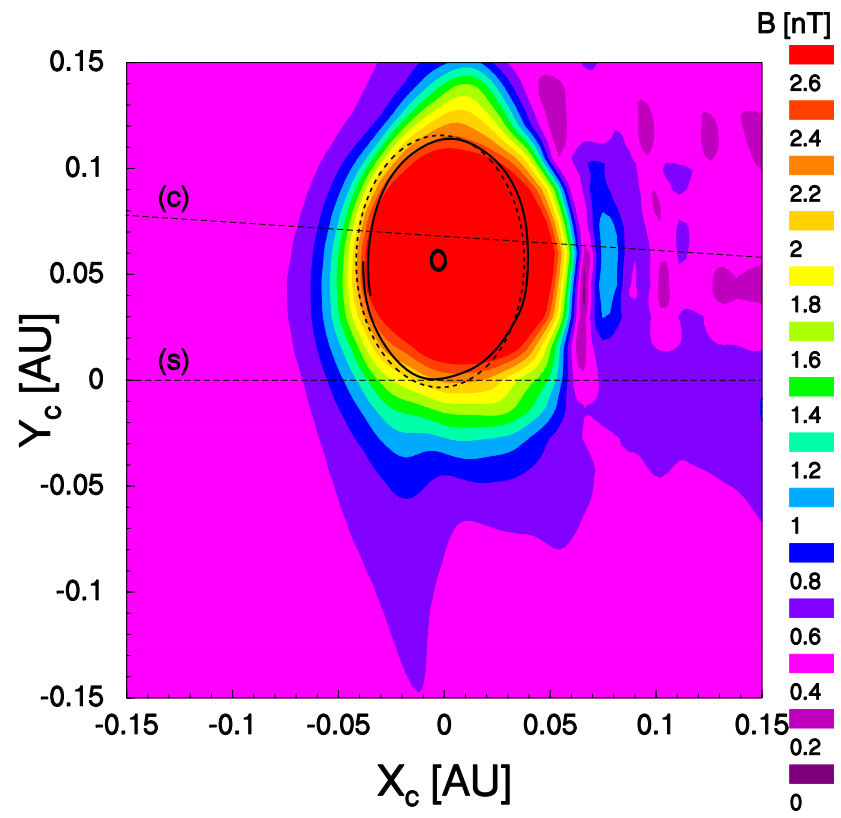

Fig. 8. Cross section through the flux rope around the point A for a $20^{\circ}$ inclined flux rope. Outline is similar to Fig. 3.

axis orientations were determined by variance analysis of magnetic field components. This was an objective procedure, the only subjective element here is an estimate of boundary crossings from "observed" magnetic field and plasma temporal profiles. Table 1 shows that variance analysis yields a good determination of the axis orientation for central crossings. It gives mixed results for side crossings. The determination of the impact factor is not reliable. Oblateness can be roughly estimated from magnetic field magnitude profiles. Oblateness does not significantly affect fits of profiles of magnetic field components.

The problem is to guess if a crossing is sufficiently close to the axis. Our simulated flux ropes have a quite regular shape, close to circular or elliptical cross sections. But if the cloud shape is distorted by its evolution in the solar corona or by dynamical interactions in a structured solar wind, the determined parameters will not be mostly reliable. So even if the fits of profiles are quite good, the determined parameters must be treated with caution. So our findings are in accord with experience gathered by Riley et al. (2004) from 2.5-D simulations.

The reason for the shape difference in our results and those from the Riley et al. (2004) paper are initial conditions. In the latter case, the flux ropes transforms into a very oblate shape during its propagation in the solar corona and exits it as an extremely oblate structure (cf. Riley and Crooker, 2004). Our simulations starts at the outer corona with a circular flux rope. Changes in its shape during propagation in the inner heliosphere are not so significant (cf. Vandas et al., 1995). If 

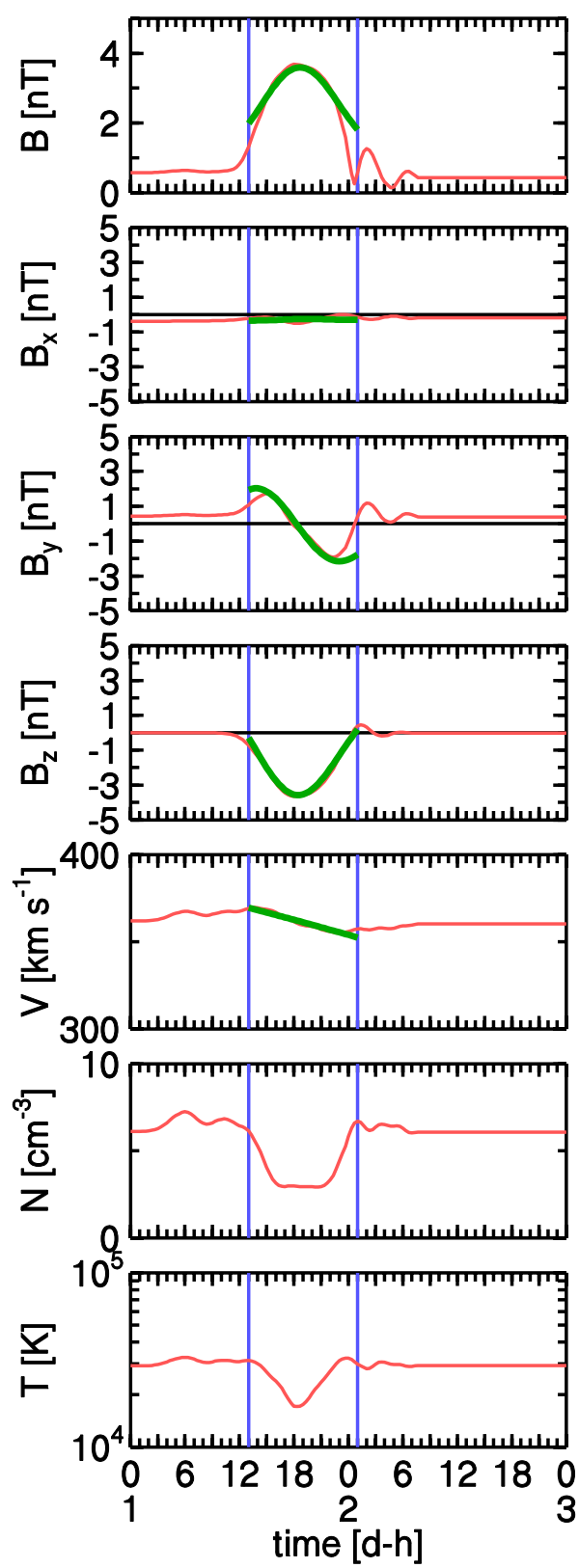

Fig. 9. Simulated time profiles at the point $\mathrm{A}$ for a flux rope inclined $90^{\circ}$ to the ecliptic plane. The layout is similar to Fig. 2.

a flux rope is close to circular at the outer corona, then it has a moderate oblateness at $1 \mathrm{AU}$. If it is pancake-shaped, then it preserves this shape. As real magnetic cloud shapes are an open question, our simulation results offer an alternative for tests of fitting procedures.

Acknowledgements. This work was supported by the project 205/09/0170 from GA ČR and PECS contract 98068 from the European Space Agency. We also acknowledge support from the Czech-US collaboration in science and technology (ME09032) provided by MŠMT ČR.
Topical Editor R. Forsyth thanks M. Hidalgo and another anonymous referee for their help in evaluating this paper.

\section{References}

Burlaga, L. F.: Magnetic clouds and force-free fields with constant alpha, J. Geophys. Res., 93, 7217-7224, 1988.

Chané, E., van der Holst, B., Jacobs, C., Poedts, S., and Kimpe, D.: Inverse and normal coronal mass ejections: evolution up to $1 \mathrm{AU}$, Astron. Astrophys., 447, 727-733, 2006.

Dasso, S., Mandrini, C. H., and Démoulin, P.: A new modelindependent method to compute magnetic helicity in magnetic clouds, Astron. Astrophys., 455, 349-359, 2006.

Hidalgo, M. A., Medina, C. C. J., and Vinas, A. F.: A new model for the magnetic topology of magnetic clouds in the solar wind, Solar Phys., 194, 165-174, 2000.

Hidalgo, M. A., Nieves-Chinchilla, T., and Cid, C.: Elliptical crosssection model for the magnetic topology of magnetic clouds, Geophys. Res. Lett., 29(13), 1637, doi:10.1029/2001GL013875, 2002.

Hu, Q. and Sonnerup, B. U. Ö.: Reconstruction of magnetic flux ropes in the solar wind, Geophys. Res. Lett., 28, 467-470, 2001.

Jacobs, C., Roussev, I. I., Lugaz, N., and Poedts, S.: The internal structure of coronal mass ejections: are all regular magnetic clouds flux ropes?, Astrophys. J., 695, L171-L175, 2009.

Kilpua, E. K. J., Liewer, P. C., Farrugia, C., Luhmann, J. G., Möstl, C., Li, Y., Liu, Y., Lynch, B. J., Russell, C. T., Vourlidas, A., Acuna, M. H., Galvin, A. B., Larson, D., and Sauvaud, J. A.: Multispacecraft observations of magnetic clouds and their solar origins between 19 and 23 May 2007, Solar Phys., 254, 325-344, 2009.

Klein, L. W. and Burlaga, L. F.: Interplanetary magnetic clouds at 1 AU, J. Geophys. Res., 87, 613-624, 1982.

Lepping, R. P., Jones, J. A., and Burlaga, L. F.: Magnetic field structure of interplanetary magnetic clouds at 1 AU, J. Geophys. Res., 95, 11957-11965, 1990.

Lepping, R. P., Berdichevsky, D. B., Wu, C.-C., Szabo, A., Narock, T., Mariani, F., Lazarus, A. J., and Quivers, A. J.: A summary of WIND magnetic clouds for years 1995-2003: model-fitted parameters, associated errors and classifications, Ann. Geophys., 24, 215-245, doi:10.5194/angeo-24-215-2006, 2006.

Liu, Y., Luhmann, J. G., Huttunen, K. E. J., Lin, R. P., Bale, S. D., Russell, C. T., and Galvin, A. B.: Reconstruction of the 2007 May 22 magnetic cloud: How much can we trust the flux-rope geometry of CMEs?, Astrophys. J., 677, L133-L136, 2008.

Manchester, W. B., Gombosi, T. I., Roussev, I., De Zeeuw, D. L., Sokolov, I. V., Powell, K. G., Tóth, G., and Opher, M., Threedimensional MHD simulation of a flux rope driven CME, J. Geophys. Res., 109, A01102, doi:10.1029/2002JA009672, 2004.

Marubashi, K.: Structure of the interplanetary magnetic clouds and their solar origins, Adv. Space Res., 6(6), 335-338, 1986.

Marubashi, K.: Interplanetary magnetic flux ropes and solar filaments, in: Coronal Mass Ejections, Geophys. Monogr. Ser., 99, edited by: Crooker, N., Joselyn, J., and Feyman, J., AGU, Washington, D. C., pp. 147-156, 1997.

Marubashi, K. and Lepping, R. P.: Long-duration magnetic clouds: a comparison of analyses using torus- and cylindershaped flux rope models, Ann. Geophys., 25, 2453-2477, doi:10.5194/angeo-25-2453-2007, 2007. 
Möstl, C., Miklenic, C., Farrugia, C. J., Temmer, M., Veronig, A., Galvin, A. B., Vršnak, B., and Biernat, H. K.: Two-spacecraft reconstruction of a magnetic cloud and comparison to its solar source, Ann. Geophys., 26, 3139-3152, doi:10.5194/angeo-263139-2008, 2008.

Mulligan, T. and Russell, C. T.: Multispacecraft modeling of the flux rope structure of interplanetary coronal mass ejections: Cylindrically symmetric versus nonsymmetric topologies, J. Geophys. Res., 106, 10581-10596, 2001.

Nakwacki, M. S., Dasso, S., Mandrini, C. H., and Démoulin, P.: Analysis of large scale MDH quantities in expanding magnetic clouds, J. Atmos. Solar-Terr. Phys., 70, 1318-1326, 2008.

Odstrcil, D., Linker, J. A., Lionello, R., Mikic, Z., Riley, P., Pizzo, V. J., and Luhmann, J. G.: Merging of coronal and heliospheric numerical two-dimensional MHD models, J. Geophys. Res., 107(A12), 1493, doi:10.1029/2002JA009334, 2002.

Riley, P. and Crooker, N. U.: Kinematic treatment of coronal mass ejection evolution in the solar wind, Astrophys. J., 600, 10351042, 2004.

Riley, P., Linker, J. A., Lionello, R., Mikic, Z., Odstrcil, D., Hidalgo, M. A., Cid, C., Hu, Q., Lepping, R. P., and Rees, A.: Fitting flux ropes to a global MHD solution: a comparison of techniques, J. Atmos. Solar-Terr. Phys., 66, 1321-1331, 2004.
Vandas, M., Fischer, S., Dryer, M., Smith, Z., and Detman, T.: Simulation of magnetic cloud propagation in the inner heliosphere in two-dimensions, 1, A loop perpendicular to the ecliptic plane, J. Geophys. Res., 100, 12285-12292, 1995.

Vandas, M., Odstrčil, D., and Watari, S.: Three dimensional MHD simulation of a loop-like magnetic cloud in the solar wind, J. Geophys. Res., 107(A9), 1236, doi:10.1029/2001JA005068, 2002.

Vandas, M., Watari, S., and Geranios, A.: Comparison of simulated and observed interplanetary flux ropes, in: Solar Wind Ten, edited by: M. Velli, R. Bruno and F. Malara, AIP Conf. Proc., 679, AIP, Melville, New York, pp. 691-694, 2003.

Vandas, M., Romashets, E., and Watari, S.: Magnetic clouds of oblate shapes, Planet. Space Sci., 53, 19-24, 2005.

Vandas, M., Romashets, E. P., Watari, S., Geranios, A., Antoniadou, E., and Zacharopoulou, O.: Comparison of force-free flux rope models with observations of magnetic clouds, Adv. Space Res., 38, 441-446, 2006. 